\title{
Dissolution of spoken language in primary progressive aphasia
}

\author{
MARGARET A. ROGERS \\ and NANCY B. ALARCON \\ Department of Speech and Hearing Sciences, University of Washington, \\ Seattle, Washington, USA
}

\begin{abstract}
The dissolution of speech and language was investigated over a 4 year period in a male with an 8 year history of isolated speech and language deterioration exhibiting a non-fluent profile of primary progressive aphasia (PPA). The course of his communication impairment began with apraxia of speech and difficulty accessing word form (e.g. as evidenced by frequent 'tip-of-thetongue' errors that typically contained correct production of word onsets or first syllables but not whole words). Ultimately all aspects of oral language use deteriorated until the individual became non-vocal. His ability to communicate through non-verbal modalities remained intact. Results of repeated administrations of standardized tests and analyses of connected speech obtained over 2 years were compared. The ability of various assessment probes to substantiate clinical impressions of noticeable declines in expressive language use was examined. It was concluded that the most sensitive indices of spoken language dissolution are likely to be measures derived from connected speech. Unlike level-specific subtests (i.e. subtests that are designed to probe relatively isolated stages of processing such as word retrieval apart from syntactic formulation), the requisite simultaneity of processing across levels during connected speech taxes the aphasic's entire language processing system. Three principles guided the approach to management: (i) anticipatory implementation of treatment goals; (ii) therapy is dyad oriented; and (iii) therapy is directed at the level of the disability, defined as the limitations to perform specific functions within a natural context The cornerstone of this approach, labelled proactive management, lies in the concept that the goals are formulated and implemented in anticipation of future declines so that the patient with PPA is prepared to maximize communication effectiveness at every stage despite the relentless progression of the disease.
\end{abstract}

\section{Introduction}

Primary progressive aphasia (PPA) is a diagnostic term that refers to the progressive deterioration of speech and language abilities concomitant with longterm (i.e. at least 2 years) preservation of other cognitive functions such as memory, reasoning, and visuospatial skills (Mesulam and Weintraub 1992). The onset and progression of this clinical syndrome are not associated with vascular, neoplastic, metabolic, or infectious disease (Duffy and Petersen 1992). PPA is distinct from dementing disorders, at least in the initial years, because in addition to preserved

Address correspondence to: Margaret A. Rogers Ph.D., Department of Speech and Hearing Sciences, University of Washington, 1417 NE 42nd Street, Seattle, WA 98105-6246, USA. 
memory abilities, individuals with PPA retain appropriate insight and judgement and are able to perform activities of daily living independently (Weintraub et al. 1990). Other symptoms of dementia not observed in PPA include personality changes, difficulty managing finances, disorientation, and denial of deficits (Duffy and Petersen 1992). Occupational disabilities are frequently a concern for patients with PPA, but unlike patients with dementia, these concerns stem exclusively from their declining abilities to perform verbally demanding activities. Thus, at least in the initial stages of PPA, the focal nature of the deficits, affecting only the areas of speech and language, clearly distinguish this population from patients with more generalized cognitive involvement.

Whereas the initial presentation of patients with PPA may be distinguishable from patients with dementia, many eventually exhibit generalized cognitive decline. Though estimates vary, as many as half exhibit more widespread cognitive decline within 5 years of the onset of speech and language deterioration (Duffy and Petersen 1992, Mesulam and Weintraub 1992). A review of the literature, summarizing 43 papers published from 1982 to 1997 (Rogers and Alarcon 1997), revealed that approximately $40 \%$ of 136 cases surveyed exhibited dementia. Rogers and Alarcon cautioned that demographic information derived from reviews of the literature should be interpreted circumspectly, however. The high degree of inconsistency regarding how many years post-onset patients happen to be at the time an article is published may confound data concerning the time course of the disease. For example, Rogers and Alarcon further reported that, when only the longitudinal cases were examined, the estimate of the proportion of cases exhibiting dementia was reduced to approximately $28 \%$. In the article containing the original labelling of this clinical syndrome, Mesulam (1982) reported that two of the six patients studied had gone more than 10 years with isolated language symptoms (i.e. had not exhibited dementia). Kempler et al. (1990) described one patient with a 20 year history of progressive aphasia without dementia. Thus despite the fact that demographic information concerning PPA has not been collected uniformly, it is reasonable to conclude that many individuals with PPA live many years without developing dementia, whereas others do not. Whether there are clinical symptoms which have prognostic value with regard to this issue remains a matter of inquiry.

The need for detailed longitudinal descriptions of the speech and language decline in PPA is indicated given

(1) the observation that the profile of language dysfunction may change over the course of the disease (e.g. Scheltens et al. 1994);

(2) that there has been speculation concerning purported prognostic value of language symptomatology with respect to future cognitive status (e.g. Snowden et al. 1992, Duffy and Petersen 1992); and

(3) that through longitudinal description, potential patterns of deterioration may be identified thereby facilitating the development of proactive management approaches.

Surprisingly, Rogers and Alarcon (1997) found only 23 studies that included longitudinal data of the 43 PPA articles reviewed. Assessment and management of the communication disability in PPA are similarly under-reported; Rogers and Alarcon (1997) reported that only four of the 43 articles surveyed addressed management. More than half of the 23 longitudinal studies focused on radiological and neuropsychological findings with only seven studies providing detailed 
descriptions of the decline in speech and language functions. The present investigation was designed to address this need for longitudinal descriptions of PPA by describing the long-term course of decline in a patient with an 8 year history of isolated speech and language symptoms with a non-fluent profile of progressive aphasia. The assessment and management approaches implemented over a 4 year period for this patient were also evaluated. Longitudinal description of this patient's speech and language assessment and treatment was completed for three reasons:

(1) to document the longitudinal course of isolated speech and language decline in an individual with an 8 year history of a non-fluent PPA;

(2) to describe effective assessment and management approaches implemented over a 4 year period; and

(3) to evaluate and recommend a framework for the future investigation of speech and language dissolution in PPA.

\section{Case report}

O.D. is a right-handed male, who was 69 years old at the onset of speech and language symptoms. He is a retired electrical engineer with a Masters degree in electrical engineering. O.D.'s significant medical history includes a mild head injury in 1945, well controlled hypertension, and ischaemic heart disease with stable angina. Angiography performed in 1988 revealed single-vessel, right coronary artery disease; angioplasty was performed in October of 1992. O.D. has a bilateral, severe sensorineural hearing loss above $3000 \mathrm{kHz}$. Other medical history for this patient is unremarkable.

At the onset of speech and language impairment in September of 1989, O.D. noticed that he was 'stumbling with multisyllabic words' such as producing 'pence fost' for 'fence post.' A month later, he discussed this problem with his primary care physician. At a 1 year follow-up appointment with his primary care physician, his speech and language difficulties were more prevalent. Initial imaging findings (head CT and MRI scan) were unremarkable. Results of neuropsychological testing performed in January 1991 were normal. On the Wechsler Adult Intelligence Scale-Revised (WAIS-R, Wechsler 1981), O.D. achieved a verbal IQ of 119, a performance IQ of 124, and a full scale IQ of 122. His age-adjusted performance on each part of the Trail-Making Test (Reitan and Wolfson 1985), which assesses attentional capacity, was also within normal limits. O.D. performed at the 89th percentile for immediate recall as assessed using the Wecbsler Memory Scale-Revised (WMS-R, Wechsler 1987) and at the 97th percentile for delayed recall. His immediate and delayed recall of the Rey Complex Figure (Rey 1964) was in the 90th percentile range. Repeat neuropsychological testing over the next 5 years showed no remarkable changes from this initial testing battery, except for the patient's performance on tests requiring intact verbal skills. In March 1991, O.D.'s neurologist diagnosed these symptoms as arising from an 'isolated primary degenerative aphasia'. In May 1992, (approximately 30 months post-onset of speech and language symptoms) a second MRI scan revealed marginal widening of the left sylvian fissure. A third MRI scan performed in February 1995, almost 3 years later, revealed two small foci of increased T2 signal: one in the left globus pallidus and a second in the region of the left superior temporal gyrus. Ventricles, sulci, and cisterns were observed to be normal; no midline shift was noted. 


\section{Initial presentation (1993)}

O.D. began treatment at the University of Washington Speech and Hearing Clinic in January 1993. He described his problem then as 'knowing the word but unable to bring the word up' and that he could not 'hear the word in his head' prior to attempting production. Administration of the Boston Diagnostic Aphasia Examination (BDAE, Goodglass and Kaplan 1983) revealed a non-fluent profile with reduced phrase length (four words), reduced articulatory agility, limited grammatical form, numerous paraphasias in running speech, repetition of highprobability sentences moderately impaired (BDAE scale rating of 2), word-finding difficulties (produced mostly content words with a high proportion of literal paraphasias), and intact auditory comprehension (BDAE percentile score of 90).

O.D.'s initial presentation in our clinic was most remarkable for his apraxia of speech (AOS), which was judged to be moderately severe at that time. His speech was characterized by laboured difficulty in articulation with delayed initiation of speech, especially with multisyllabic words, short phrases fragmented with fillers, and frequent intersyllabic intrusions of schwas. Multiple attempts to produce a target word resulted in varying errors including both vowel and consonant substitutions, simplifications, and distortions. O.D. exhibited improved performance when the target was modelled, when he read the word, and when he learned to produce multisyllabic targets one syllable at a time (i.e. syllable segregation). This profile of symptoms was judged to be consistent with a diagnosis of AOS by three American Speech and Hearing Association (ASHA) certified speech-language pathologists. O.D. did not exhibit symptoms consistent with a diagnosis of oral-facial apraxia at the time of initial testing nor at subsequent testing dates through to May 1997. Periodic structural-functional oral mechanism examinations were administered from January 1993 to May 1997. No evidence of muscular weakness, spasticity, or structural abnormalities were observed at any of the testing dates.

\section{Speech, language, and cognition (1993 to 1997)}

Over the course of the 4 year period during which O.D. received services in our clinic, all aspects of his spoken language production declined. Auditory comprehension, reading, and writing were somewhat more resistant to the disease process than verbal expression, but eventually these declined as well. Figure 1 depicts this decline across these four modalities as represented by O.D.'s performance on the Boston Naming Test (BNT, Kaplan et al. 1983), the Written Spelling subtest of the Minnesota Test for Differential Diagnosis of Aphasia (MTDDA, Schuell 1973), the Sequential Commands subtest and the Reading Commands subtest of the Western Aphasia Battery (WAB, Kertesz 1982). The asynchronous onset of impairment in naming, spelling, auditory comprehension, and reading comprehension was of particular interest in this figure. The order of decline depicted by the results of standardized subtests in figure 1 was consistent with both the client's observations and clinical impressions. As can be seen in this figure, O.D.'s expressive and receptive decline was substantial over the 4 year period, with expressive abilities declining ahead of receptive abilities. The declines observed in O.D.'s writing reflect his difficulty in spelling words correctly in their entirety. Despite the fact that his MTDDA Written Spelling scores declined early 


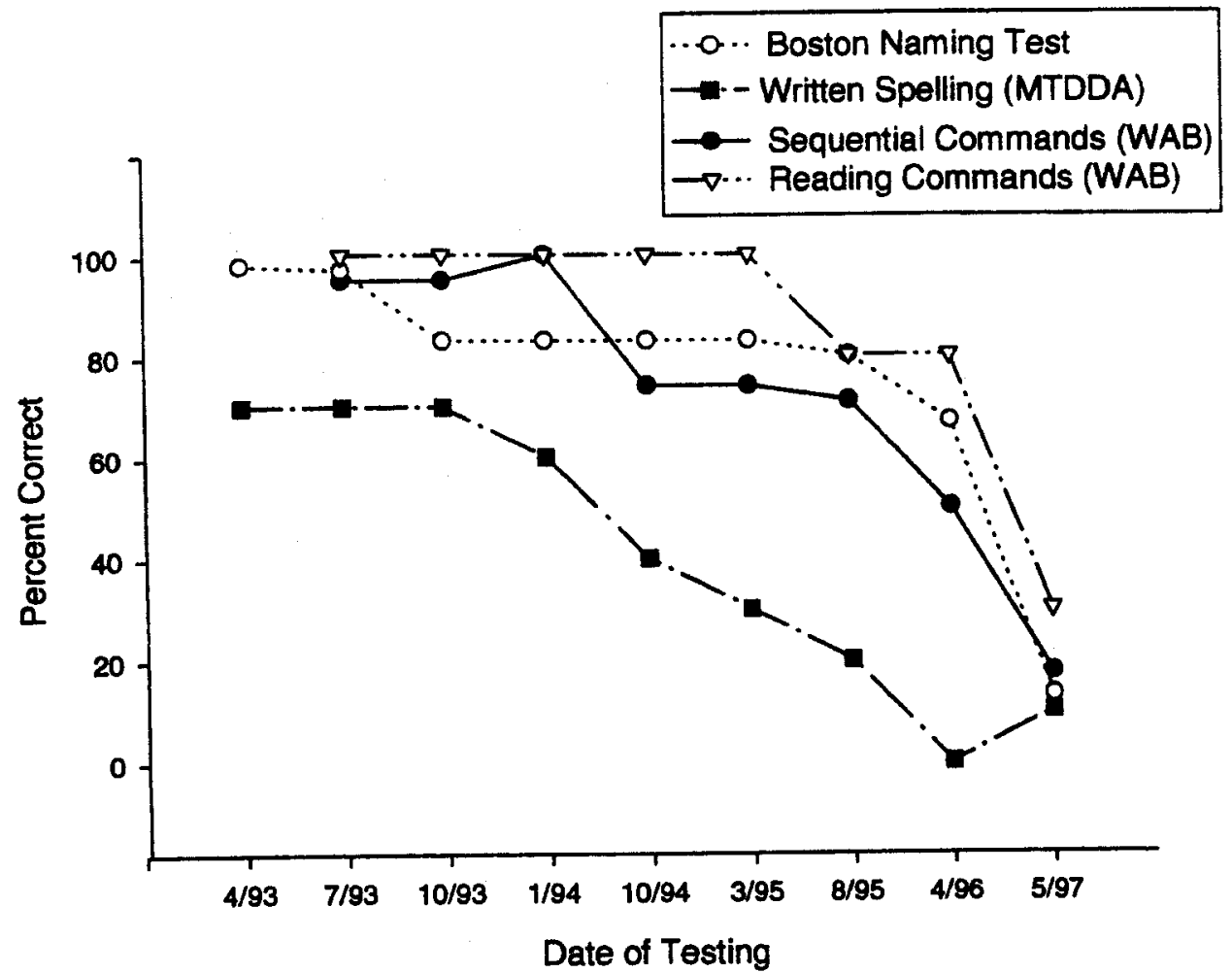

Figure 1. Selected results of speech and language assessment from 1993 to 1997. Testing in the areas of auditory comprehension, verbal expression, reading comprehension, and writing are shown.

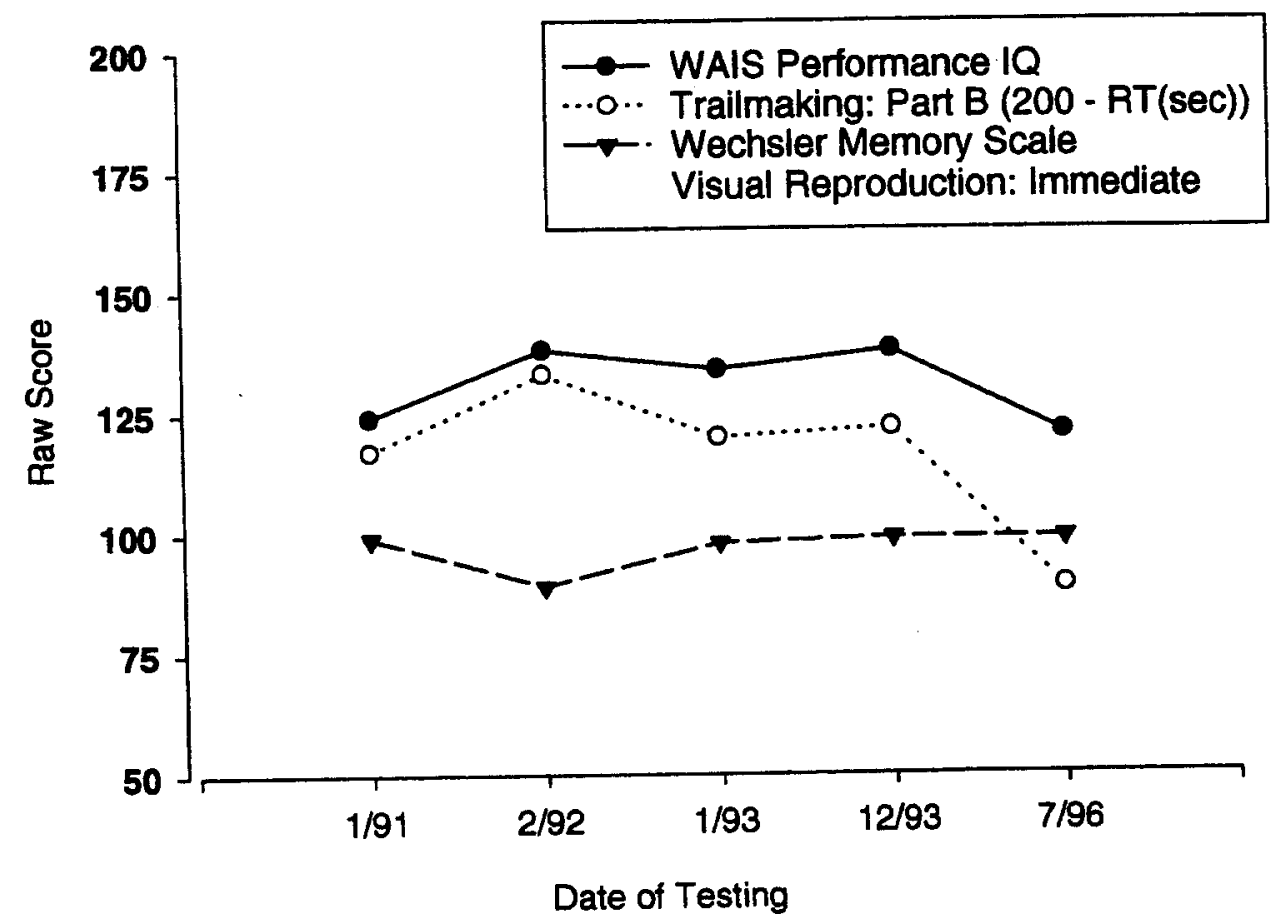

Figure 2. Selected results of neuropsychological testing from 1991 to 1996.

and rapidly, O.D. continued to write single words as a primary communication tool through to August 1997 (i.e. until the time of writing this publication). Though these written words were typically incomplete, they were generally accurate enough to be comprehended most of the time. 


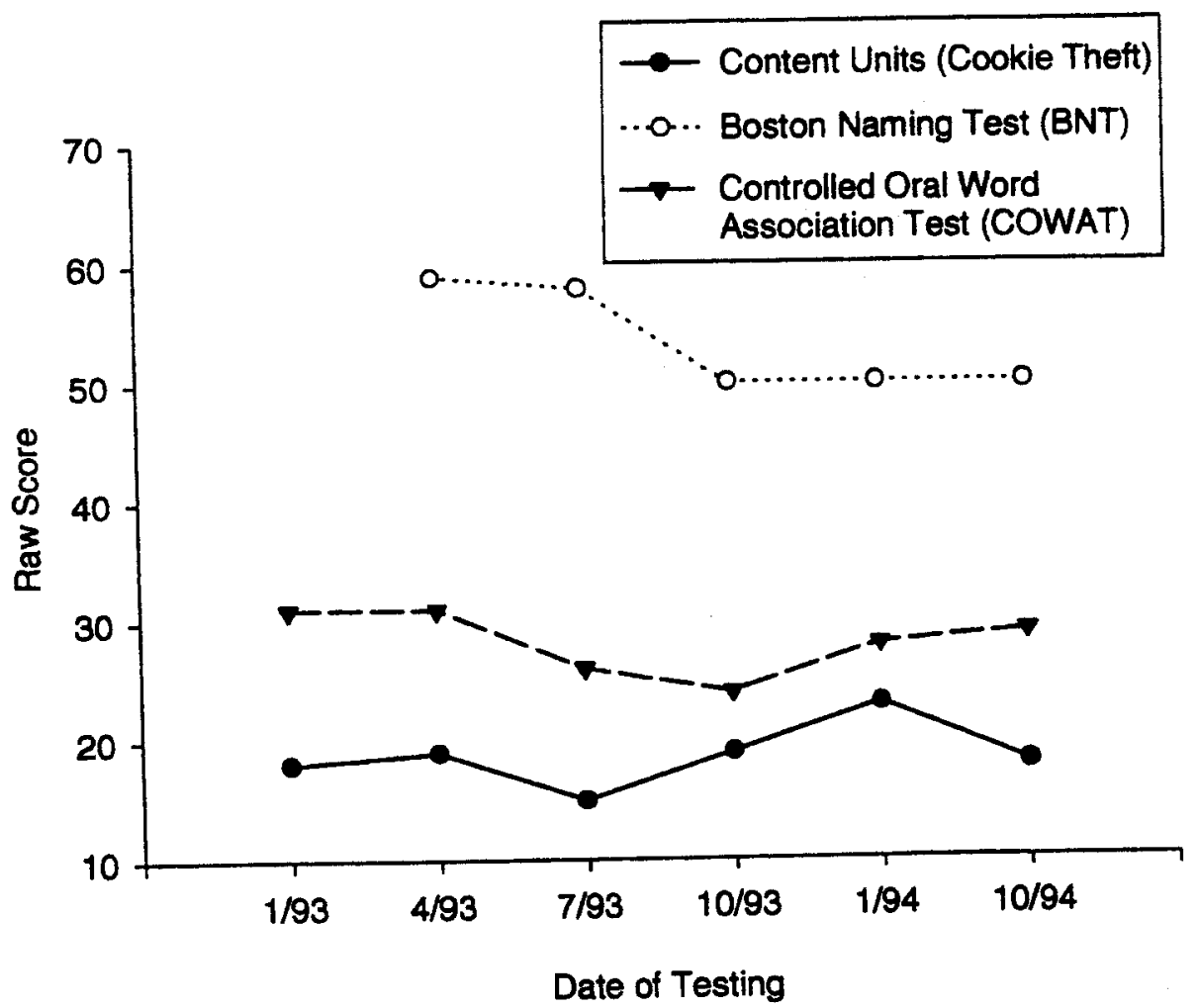

Figure 3. Selected results of tests of verbal expression from January 1993 to October 1994.

Figure 2 shows the results of selected cognitive testing from 1991 to 1996. In sharp contrast to the results of speech and language testing, these tests of cognitive status revealed an unchanging cognitive profile over the period studied. In addition to the WAIS performance IQ and Wechsler Memory shown in figure 2, O.D.'s immediate and delayed recall of the Rey Complex Figure, his performance on the Trail-Making Test and the Halstead-Reitan Category Test (Reitan and Wolfson 1985) were found to be within normal limits in July 1996.

\section{January 1993 to October 1994}

Whereas clinical impressions were generally consistent with the long-term trends obtained from standardized tests of speech and language function, short-term declines observed clinically were not reflected by these same tests. Between January 1993 and October 1994, O.D. began to shift from adequate verbal communication to greater reliance on non-verbal communication. His oral expressive abilities declined rapidly during this time according to his family, his clinicians, and selfreport. Contrary to these impressions, standardized measures of expressive language failed to indicate a decline in his ability to use oral language over this 2 year period (see figure 3). Figure 3 shows the results of selected tests of verbal expression from January 1993 to October 1994. The results of three measures are shown: Content Unit Identification (Yorkston and Beukelman 1980) with the 'Cookie Theft Picture Description Task' from the BDAE; the BNT; and the Controlled Oral Word Association Test (COWAT, Benton and Hamsher 1978). O.D. performed within the range for normal adults on Content Unit Identification for all six testing sessions shown in figure 3 . On the BNT and COWAT, his test scores over this 2 year period remained essentially unchanged and consistently 


\section{Discourse Analysis - Speech Rate}

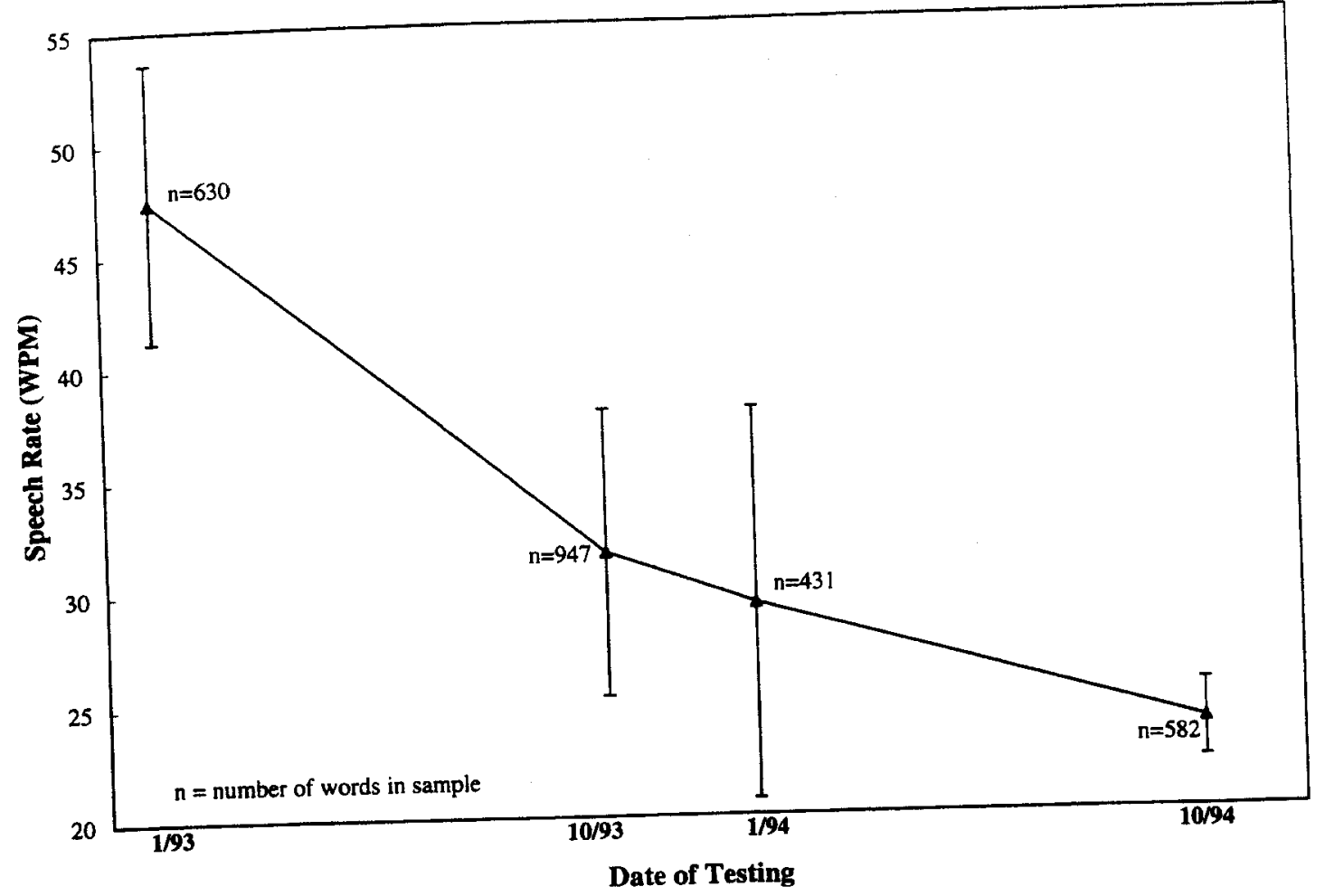

Figure 4. Results of speech rate analyses performed on spoken discourse samples obtained on four dates between January 1993 and October 1994.

placed him in the 'low normal' range. Despite the relative stability of these three measures, O.D.'s expressive language became increasingly telegraphic during this period and his use of non-verbal communication (e.g. communication book, writing, drawing) became more frequent.

\section{Discourse analyses}

More sensitive indices of the rate of language dissolution observed during the period from January 1993 to October 1994 were derived by analysis of connected speech. The three elicitation contexts used were based on the procedures used by Nicholas and Brookshire (1993): picture description (the 'cookie theft' picture from the BDAE, Goodglass and Kaplan 1983), the two single-frame pictures and two six-frame pictures from Nicholas and Brookshire (1993); requests for procedural information ('Tell me how you would go about doing dishes by hand' 'Tell me how you would go about writing and sending a letter'); and requests for personal information ('Tell me what you do on Sundays' 'Tell me where you live and describe it to me'). These three types of speech samples were elicited twice a year over a 2 year period. The three samples were combined to comprise the final data source which consisted of at least 400 words for each of the sampling dates. The analyses included speaking rate (in words per minute, WPM), the mean length of utterance in morphemes (MLU), and per cent correct information units (\%CIUs, Nicholas and Brookshire 1993). The measure of CIUs involves a count of intelligible words that are accurate, relevant, and informative relative to the eliciting stimulus though they need not have been produced in a grammatically accurate manner. Each CIU consists of a single word and the \%CIUs is relative to 


\section{Discourse Analysis - Utterance Length}

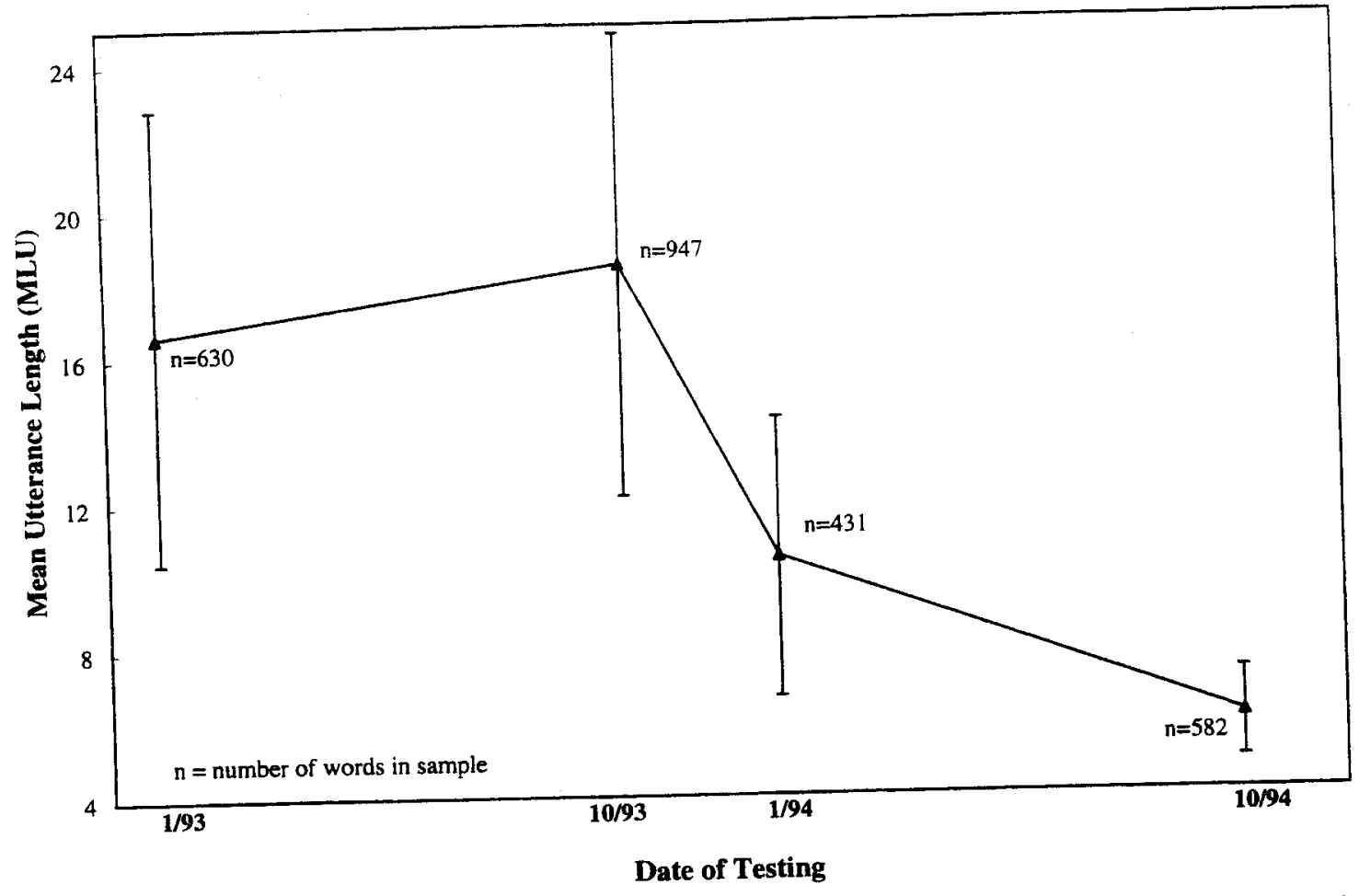

Figure 5. Results of mean length of utterance analyses performed on spoken discourse samples obtained on four dates between January 1993 and October 1994.

the total number of intelligible words produced. The results of the discourse analyses are presented in figure 4 (speaking rate), figure 5 (MLU), and figure 6 (\%CIUs). The shared (i.e. by O.D. and the clinicians) subjective impression of sharply declining expressive abilities was substantiated by each of these measures. The most dramatic change in speech rate occurred between the first and second sampling dates: January 1993 and October 1993. The sharpest decline in utterance length occurred between the second and third sampling dates: October 1993 and January 1994. The most noticeable decline in \%CIU was the drop below the normal range which occurred between the third and the fourth sampling dates: January 1994 and October 1994. Thus, there was considerable asynchrony among these measures with respect to the period that evinced the greatest decline in function.

The Appendix contains two speech samples, one obtained in October 1993 and the second in October 1994. Both samples were elicited by asking O.D. to describe the 'Birthday Party' picture from Nicholas and Brookshire (1993). The Appendix displays the number of morphemes in each sample, intelligible words, WPM, unintelligible words, non-word fillers, CIUs, non-CIUs (i.e. intelligible words that were not CIUs), and \%CIUs. The most notable contrast between these two samples is that the percentage of non-CIUs increased from $23 \%$ of intelligible words to $52 \%$ of intelligible words over the two samples. Conversely, \%CIUs decreased from $77 \%$ to $48 \%$ from October 1993 to October 1994. The number of CIUs per minute also declined from 29 to 10 , as did WPM, which declined from 37 to 21 over these two samples. Other measures did not change as noticeably. The decline observed in \%CIUs during this period stemmed mainly from the increase of filler words including repetitions and frequent use of the words 'and', 'the', and 'to'. 
Discourse Analysis - Correct Information

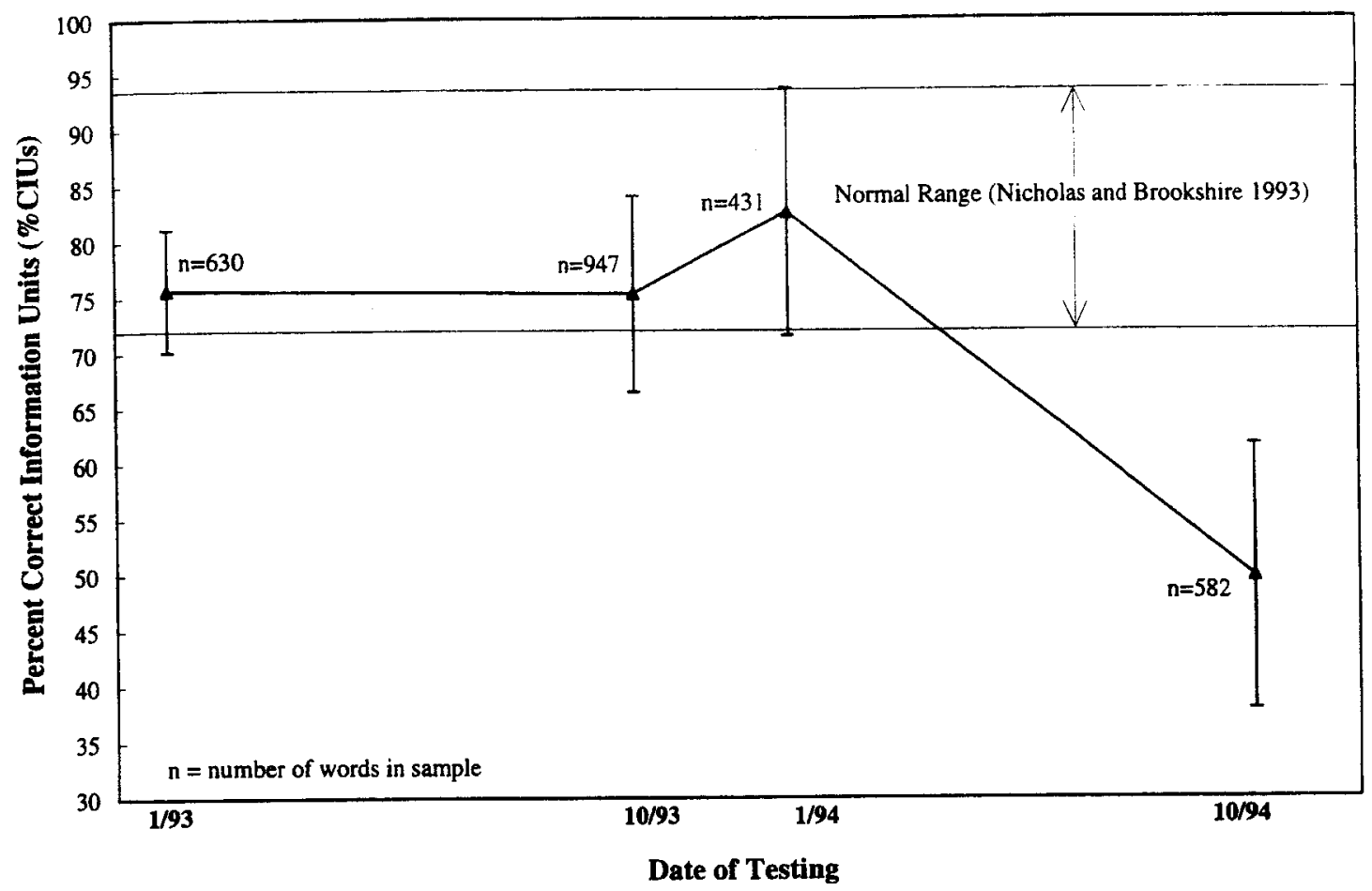

Figure 6. Results of per cent correct information units performed on spoken discourse samples obtained on four dates between January 1993 and October 1994.

\section{Accounting for asyncbronous declines}

The source of the asynchronous declines across speaking rate, MLU, and \%CIUs observed might be accounted for by hypothesizing asynchronous declines among speech and language subsystems (e.g. motor programming, syntactic formulation, phonologic encoding). For example, the initial decline in speaking rate could be attributed to increased difficulty with motor programming; the subsequent decline in MLU may have reflected increased syntactic formulation dysfunction; and the latest appearing decline in \%CIUs may have resulted from increased difficulty accessing word form compounded by further declines in other areas. Support for an account based on changes occurring at the level of the impairment requires correlative data to demonstrate the covariation of these speech and language deficits with other tests, specifically sensitive to each of these processing stages. Unfortunately, correlative data of this nature were not obtained in a systematic manner with O.D. In an effort to suggest a framework for the future investigation of speech and language dissolution in PPA, it is recommended that level-specific probes (e.g. probes of articulation, motor programming, phonologic encoding, grammatical encoding) as well as measures derived from connected speech (e.g. WPM, MLU, \%CIUs) be administered at regular intervals.

An alternative explanation of the asynchronous decline of these three discourse measures is that O.D. accommodated the increasing severity of his oral-verbal communication impairment by successive compensatory adjustments. Accordingly, one might conjecture that the simplest and most effective compensation to declining oral-verbal communication abilities is merely to slow down. As the degenerative process continues and the ameliorative effect of slower rate diminishes, one might begin to decrease utterance length. Lastly, as utterance 
length becomes maximally restricted, the \% CIU would necessarily decline since many propositions are difficult to convey with an utterance length of only two to four morphemes. Though this hypothesis is plausible, close inspection of the data suggests that it has weaknesses. For example, speaking rate declined further after utterance length declined sharply. Since further rate compensations were possible when utterance length was being markedly reduced, it was not the case that utterance length changes were implemented only when the benefits of rate reduction had 'run out.' Of course, it may be that patients with degenerative communication impairments do not adopt compensatory strategies such as these in a unitary, quantal manner, exhausting the utility of one strategy before adopting the next. Thus, the issue of whether the asynchrony in the decline of speaking rate, utterance length, and \%CIU reflects the dissociated decline of specific processing components or the order in which compensatory strategies are implemented remain equivocal. It is likely that the pattern observed reflects a combination of the impairment worsening and O.D.'s compensatory adjustment to these declines.

\section{Indices sensitive to spoken language dissolution}

A puzzling result of the present investigation was the insensitivity of content unit analysis, the $\mathrm{BNT}$, and the COWAT to the declines evident in speaking rate, utterance length, and \%CIU. The former measures may not have reflected the processing demands inherent in the production of connected speech. The content unit analysis, the BNT, and the COWAT are minimal measures of production and as such, adequate performance on these tasks does not require integrated processing of semantic, syntactic, and phonologic information. Furthermore, these tasks involve minimal motor programming demands, as adequate performance on these measures can be achieved even if the speaker formulates words one at a time. Speaking rate, utterance length, and \%CIU, on the other hand, are sensitive to phrase-length properties of speech. These discourse measures are probably more sensitive to gradual deterioration of spoken language production because they entail integration of linguistic processes (i.e. semantic, syntactic, phonologic, and motor encoding operations).

In summary, it appears that single word production tasks failed to tax spoken language processing to the degree that connected speech did. The demand for integrated processing across all aspects of spoken language production (e.g. semantic, syntactic, phonologic, motor programming) is inherently less for tasks that only require single word production. It is hypothesized that the excessive demands imposed by the integrated processing requirements of connected speech may have exceeded the capacity of O.D.'s language production system. Thus, even though the single word production tasks were insensitive to O.D.'s deteriorating speech, his ability to formulate connected discourse more accurately reflected this marked decline. The present results suggest that the most sensitive indices of spoken language dissolution are likely to be measures derived from connected speech.

In general, the approach to long-term assessment of speech and language dissolution in PPA being advocated would include a range of level-specific probes such that the status of speech and language subsystems (e.g. articulation, motor programming, phonologic encoding, grammatical encoding) might be monitored. Probes should be selected and periodically re-administered to sample both affected 
and unaffected aspects of speech and language. This monitoring facilitates detection of changes in previously unaffected areas and identifies areas of relative strength, which may be exploited to compensate for areas of weakness. In addition to monitoring the status of speech and language subsystems, measures that probe the ability to perform tasks requiring integrated processing across levels of language production (i.e. measures obtained from spoken discourse) are also advocated as they appear to be more sensitive to relatively subtle changes observed in the short term (e.g. over the course of a few months).

\section{Proactive management: effective intervention in PPA}

The philosophy of intervention for this patient's PPA is best described by the term proactive management. The cornerstone of this approach is the formulation and implementation of treatment goals in anticipation of future declines. Figure 7 summarizes the course of O.D.'s communication impairment observed from 1993 to 1997 in the left-hand column and the course of intervention strategies implemented in the right-hand column. The initial approach included goals directed to improve or slow the decline of his word production impairments (e.g. Integral Stimulation, Rosenbek and LaPointe 1985, Wertz et al. 1984) but it was quickly apparent that these efforts were ineffectual. After 6 months, a proactive approach to management was implemented guided by the perceptions that deterioration could not be forestalled, that non-verbal modalities were declining less rapidly than speech, and that O.D.'s wife needed to adjust her approach to communication as much as O.D. needed to adjust his own. The proactive approach to the management of PPA that guided O.D.'s treatment is elucidated by the following three principles.

\section{Principle I: Anticipatory implementation of treatment goals}

The first principle of proactive management is that therapy goals should be implemented in anticipation of continued decline in communication independence. It is the downward path along the continuum of communication independence that prompts the first principle of proactive management. Specifically, as oral-verbal communication becomes increasingly less effective, more of the communication burden becomes transferred to the unimpaired partners and the individual with PPA must increasingly rely on augmentative and assistive communication (AAC). As can be seen in figure 7, the course of intervention implemented with O.D. was responsive to his need increasingly to rely on his communication partners and on non-verbal communication. His gradual transition from an 'unassisted' to an 'assisted' communicator is evident in the stages shown in figure 7. It is the degenerative nature of PPA that necessitates a different approach to intervention from that used in the treatment of 'static' aphasia. Whereas principles II and III are equally applicable to treating 'static' aphasia, it is principle I that is uniquely designed to accommodate the degenerative nature of PPA. As the communication abilities of individuals with PPA evolve away from complete independence toward requiring assistance from communication partners and augmentative techniques, the skills and tools that will enable them to maximize their communication efforts should already be in place.

Anticipating continual decline in communication independence has two 


\section{Course of Communication...}

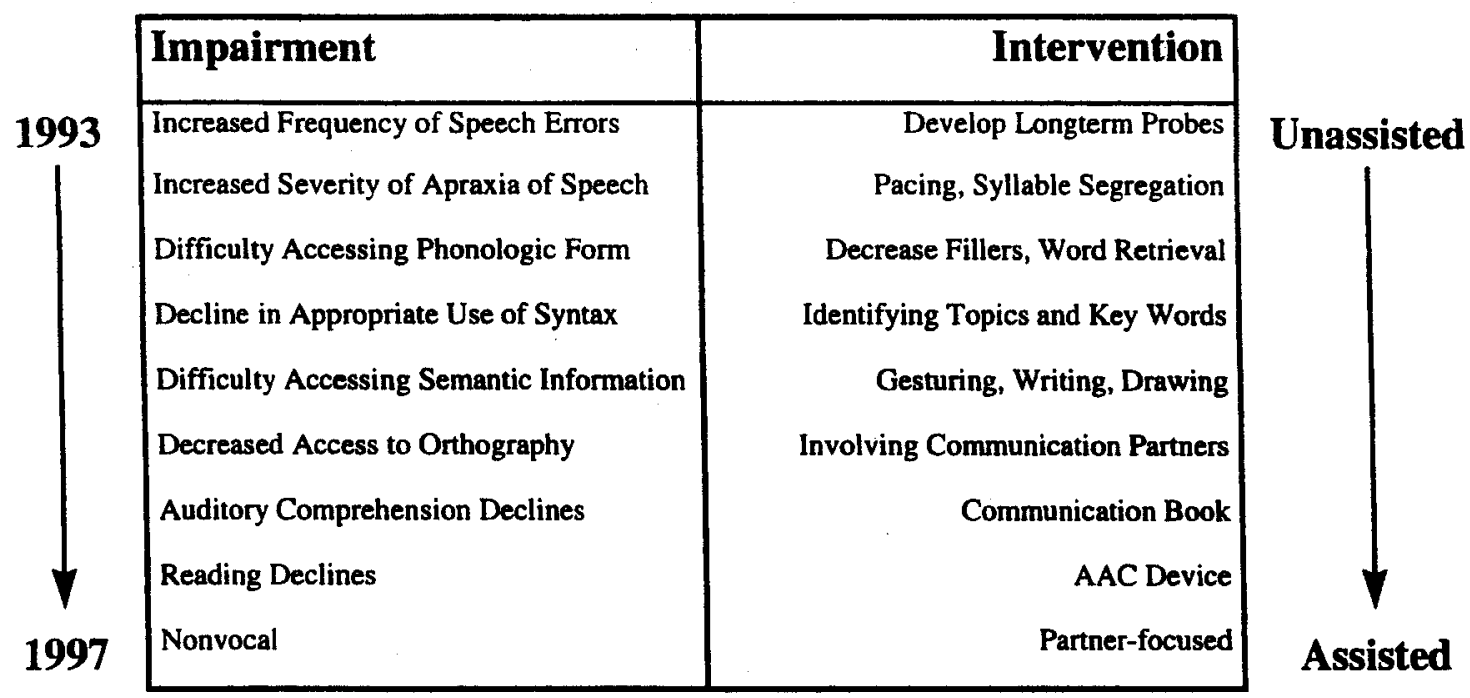

Figure 7. The course of O.D.'s speech and language decline is schematically depicted relative to the course of intervention that was proactively implemented.

important implications. The first is that compensatory techniques should be aimed at a level of impairment that is more severe than the patient's immediate status might warrant. For example, the communication notebook was introduced to O.D. at a time when he was still succeeding with oral-verbal communication. $\mathrm{He}$ had a difficult time accepting the notebook and using it for this reason. Nevertheless, it was appropriate to develop its content while O.D. could still speak and spell well enough to participate fully in its construction. The transference of responsibility for the development of the notebook to O.D. ensured that items he would be likely to use were included, that it became organized in a manner that appealed to him, and that it promoted acceptance. By the time O.D. needed the notebook, he had developed it to a degree that afforded communication on a wide range of topics. He was very familiar with its content and organization and he had already accepted his need to use it. Thus, by anticipating the course of his impairment, we were able to prepare this patient to maximize his communication effectiveness in advance of the need.

\section{Principle II: Therapy is dyad oriented}

Another implication of declining communication independence is that the communication partners of individuals with PPA play an increasingly important role in communication. For example, O.D.'s wife had to alter her discourse style to accommodate O.D.'s expressive and receptive difficulties. To accommodate his receptive difficulties, she reduced her speaking rate, reduced tangential conversational topic shifts, eliminated multiple embedded questions, and eventually, wrote key concepts and verified the accuracy of her husband's comprehension. To accommodate his expressive impairment, she developed guessing strategies to determine misarticulated or misspelled words and learned to encourage his use of multi-modality communication. Aspects of their interaction style were also addressed. O.D. and his wife developed signals for when and how she was to assist 
him. The development of mutually understood idiosyncratic gestures proved to be another useful augmentation. Examples of these included gestures to indicate past, present, and future tense, to identify specific people and places, as well as gestures to indicate emotional states. They also received guidance with respect to developing patience and ways to ameliorate communication breakdowns (e.g. identifying the breakdown, sorting clear from ambiguous information, and logical enquiry to achieve successful communication). Training the spouse began with general education concerning the use of facilitatory behaviours to communicate effectively with individuals with aphasia (e.g. simplifying language, reducing speaking rate, using and encouraging multiple modalities to communicate). These techniques were then practised in therapy with both O.D. and his wife, feedback was provided by the clinician, and self-monitoring of both facilitatory and non-facilitatory behaviours (e.g. lengthy and complex utterances, rapid speaking rate, communication relying solely on spoken language) was mastered using off-line analysis of videotaped conversations and on-line monitoring during therapy sessions. Generally, as the severity of the communication impairment increases, the treatment becomes increasingly focused on the communication partner, as the burden of communication naturally shifts to the more skilled partner. The need to involve communication partners in treatment is critical and should occur as early in the intervention as possible.

\section{Principle III: Therapy-is directed at the level of the disability}

Proactive management entails assisting the client to use areas of relative strength and stability to compensate for areas that are relatively weak or declining. Neither restoration nor maintenance of function is advocated as an appropriate goal for patients with PPA. Rather, aspects of communication function that are relatively intact (i.e. the client's 'strengths') must be used to augment or circumvent areas of relative weakness. O.D.'s initial impairment arose from AOS and difficulty accessing word form. During this period, O.D. communicated much the way he always had, only far less successfully and with far greater effort. The goal of therapy during this initial period was to decrease his disability by teaching him to pace (i.e. slow down and insert more pauses), to produce multisyllabic words one syllable at a time (i.e. syllable segregation), and to decrease the excessive use of non-word fillers (e.g. ' um' and 'ah'). Efforts directed toward maintenance of skills relative to speech production (e.g. Integral Stimulation) and appropriate use of syntax (e.g. practice with verb tense and question formation) did not appear to retard the deterioration process, although it is impossible to know how quickly his symptoms might have progressed without treatments. Ultimately, he became entirely nonverbal due primarily to the severity of his AOS. Currently, he uses a DynaVox 2 device (C) Sentient Systems), skilfully uses a combination of gestures, writing, and drawing, and relies heavily on a well-developed communication notebook. O.D. was encouraged to pair gestures and drawing with verbal communication (i.e. writing and speaking) during the early stages of treatment, before it was really necessary. This approach is proactive in the sense that the client has the opportunity to practice and improve communicating with gestures and drawings while the verbal modalities remain viable. Thus, by the time the verbal modalities have ceased to be functional, the client has already become accustomed to communicating using a variety of techniques in non-verbal modalities. 


\section{Summary}

This investigation describes the communicative decline of a patient with PPA. The onset of speech and language symptoms in this patient consisted primarily of AOS and impaired access to word form. Within 4 years post-onset, his symptoms became consistent with a non-fluent profile of aphasia. Six years post-onset, O.D. could still vocalize and infrequently attempted single word production, but essentially he had become non-verbal due to the severity of AOS. At 8 years postonset, O.D. continued to drive, take care of his own finances, and function independently in all aspects of his life except for communication. These factors, in concordance with the results of neuropsychological testing, affirm that O.D. exhibited isolated speech and language symptoms for an 8 year period, up to the time of this report.

It was suggested that standardized speech and language subtests may lack the sensitivity required to track the slow and complex decline in language function exhibited by individuals with PPA. These subtests do not require integrated processing across the multiple levels entailed in spoken language production. Furthermore, it has been suggested that the most sensitive indices of language dissolution may be measures obtained from discourse production precisely because this context taxes the speaker's ability to integrate simultaneously semantic, syntactic, phonologic, and motor programming operations.

Finally, a proactive management approach has been advocated based on three principles. The first principle is based on the observation that communication independence declines as the disease progresses. Therapy goals should be implemented in anticipation of continued decline in communication independence. This adjustment does not amount to undercutting prematurely the client's communication independence. Rather, this approach attempts to acclimatize the client to a variety of communicative modalities and to facilitate assistance from communication partners to maximize communicative effectiveness at every stage during the decline. The second principle is that including the communication partners of individuals with PPA early in the therapy process is critical. The dynamic and insidious nature of the communication impairment predicts that patients with PPA will eventually become quite dependent on the assistance of their conversational partners for successful communication. The third principle simply advocates directing therapy at the level of the disability, as opposed to treating the impairment, since the individual with PPA must ultimately learn to compensate for the continual deterioration of verbal communication.

\section{Acknowledgements}

This work has been supported by The Hill Family Foundation and The Schilling Fund. We would also like to acknowledge Orville and Betty Dunn as they taught us much about how to treat PPA and the value of dyad-directed therapy. We appreciate having had the opportunity to work with them and to know them.

\section{References}

Benton, A. L. and Hamsher, K. 1978, Multilingual Aphasia Examination (Iowa City: Benton Laboratory of Neuropsychology). 
Duffy, J. R. and Peterson, R. C. 1992, Major review: primary progressive aphasia. Aphasiology, 6 , $1-13$.

Goodglass, H. and Kaplan, E. 1983, The Assessment of Apbasia and Related Disorders (2nd edn) (Philadelphia: Lea \& Febiger).

Institute of Medicine 1991, Disability in America: Toward a National Agenda for Prevention (Washington, DC: National Academy Press).

Kaplan, E., Goodglass, H. and Weintraub, S. 1983, The Boston Naming Test (Philadelphia: Lea \& Febiger).

Kempler, D., Metter, E. J., Riege, W. H., Jackson, C. A., Benson, D. F. and Hanson, W. R. 1990, Slowly progressive aphasia: three cases with language, memory, CT and PET data. Journal of Neurology, Neurosurgery, and Psychiatry, 53, 987-993.

Kertesz, A. 1982, The Western Aphasia Battery (New York: Grune \& Stratton).

Mesulam, M. M. 1982, Slowly progressive aphasia without generalized dementia. Annals of Neurology, 11, 592-598.

Mesulam, M. M. and Weintraub, S. 1992, Spectrum of primary progressive aphasia. In M. N. Rossor (Ed.) Baillière's Clinical Neurology. Vol. 1, Unusual Dementias (London : Baillière Tindall), pp. 43-66.

Nicholas, L. E. and Brookshire, R. H. 1993, A system for quantifying the informativeness and efficiency of the connected speech of adults with aphasia. Journal of Speech and Hearing Research, 36, 338-345.

Reitan, H. M. and Wolfson, D. 1985, The Halstead-Reitan Neuropsychological Test Battery (Tucson: Neuropsychology Laboratory).

REy, A. 1964, L'examen Clinique en Psychologie (Paris: Presses Universitaires de France).

Rogers, M. A. and Alarcon, N. B. 1997, Assessment and management of primary progressive aphasia: A longitudinal case study over a five year period (Presentation at the 1997 Clinical Aphasiology Conference, MT) (Submitted).

Rosenbek, J. C. and LAPornte, L. L. 1985, The dysarthrias: description, diagnosis, and treatment. In D. F. Johns (Ed.) Clinical Management of Neurogenic Communicative Disorders (Boston: Little, Brown), pp. 97-152.

Scheltens, P., Ravid, R. and Kamphorst, W. 1994, Pathologic finding in a case of primary progressive aphasia. Neurology, 44, 279-282.

Schuel, H. 1973, Differential Diagnosis of Aphasia with the Minnesota Test (revised by J. W. Sefer) (Minneapolis: University of Minnesota Press).

Snowden, J. S., Neary, D., Mann, D. M. A., Goulding, P. J. and Testa, H. J. 1992, Progressive language disorder due to lobar atrophy. Annals of Neurology, 31, 174-183.

Wechsler, D. 1981, Wechsler Adult Intelligence Scale-Revised [Manual] (New York: Psychological Corporation).

Wechsier, D. 1987, Wechsler Memory Scale-Revised (San Antonio: The Psychological Corporation).

Weintraub, S., Rubin, N. and Mesulam, M. 1990, Primary progressive aphasia: longitudinal course, neuropsychological profile, and language features. Arcbives of Neurology, 47, 1329-1335.

Wertz, R. T., LaPointe, L. L. and Rosenbek, J. C. 1984, Apraxia of Speech in adults: The Disorder and its Management (New York: Grune \& Stratton).

Yorkston, K. M. and Beukelman, D. R. 1980, An analysis of connected speech samples of aphasic and normal speakers. Journal of Speech and Hearing Disorders, 45, 27-36. 


\section{Appendix}

Picture description of 'The Birthday Party' (Nicholas and Brookshire 1993) produced by O.D. in October 1993.

It's a uh start of a uh birthday party and the uh uh cake is uh uh bit uh bite uh bit and uh the uh uh dog is uh bite of the uh uh cake uh the do that and uh he was uh uh under the uh $/ \mathrm{k} \varepsilon \mathrm{d} /$ / (couch) and the uh uh mother was uh have a broom an uh uh uh (unintelligible word) like hit uh uh the dogs and the uh $\operatorname{dog}$ uh foot (unintelligible word) the tables and the uh um / kæpə/ (carpet) and the uh uh birthday uh kid was uh (unintelligible word) crying and uh uh not (unintelligible word) but uh uh (unintelligible word) and uh uh oh my uh eyes is down and uh uh two uh uh two uh uh /msnadrs / (mothers) and uh one uh boy and uh one uh uh girls is uh coming to the uh uh / parpi/ (party) and they uh uh have uh uh gifts to uh uh birth birthday kids.

(Elapsed time $=145 \mathrm{~s})$

\begin{tabular}{lcccccc}
\hline Morphemes & $\begin{array}{c}\text { Intelligible } \\
\text { words (WPM) }\end{array}$ & $\begin{array}{c}\text { Unintelligible } \\
\text { words (PM) }\end{array}$ & $\begin{array}{c}\text { Non-word } \\
\text { fillers (PM) }\end{array}$ & CIUs (PM) & $\begin{array}{c}\text { Non-CIUs } \\
(\% \text { non-CIUs) }\end{array}$ & $\%$ CIU \\
\hline 99 & $90(37)$ & $5(2)$ & $63(26)$ & $69(29)$ & $21(23 \%)$ & $77 \%$ \\
\hline
\end{tabular}

Picture description of 'The Birthday Party' (Nicholas and Brookshire 1993) produced by O.D. in October 1994.

The uh birthday day / parkas/ (party) and the uh dog uh the uh bite the uh uh cake and uh / tri/ (three) uh four uh / ketls/ (candles) the um and the uh /kat/ (cake) oh and the uh (unintelligible word) to the uh (unintelligible word) and uh the uh little uh boy was uh uh crying and the uh your uh mother's uh um broom the um (unintelligible word) the uh (unintelligible word) the uh $/ \mathrm{d} æ \mathrm{~g} /(\mathrm{dog})$ in uh in (unintelligible word) uh two uh two uh kids um one and two uh your uh mother the uh (unintelligible word) the uh the uh go the uh uh room and the uh two uh kids uh they uh /gf/ (give) the uh birthday /gxft/ (gift) um two uh pictures on the uh wall the uh /pafar/ (picture) the uh (unintelligible word) uh like uh lamp the uh tables uh the uh after the uh (unintelligible word) / nu/ (two) two uh one /wandru/ (window) and the see uh uh trees and uh /mantan/ (mountain) um the uh chairs and the uh in the uh or the uh (unintelligible word) and the uh the um one uh candles uh the uh on the uh uh table. Okay?

$($ Elapsed time $=306 \mathrm{~s})$

\begin{tabular}{lcccccc}
\hline Morphemes & $\begin{array}{c}\text { Intelligible } \\
\text { words (WPM) }\end{array}$ & $\begin{array}{c}\text { Unintelligible } \\
\text { words (PM) }\end{array}$ & $\begin{array}{c}\text { Non-word } \\
\text { fillers (PM) }\end{array}$ & CIUs (PM) & $\begin{array}{c}\text { Non-CIUs } \\
(\% \text { non-CIUs) }\end{array}$ & $\%$ CIU \\
\hline 127 & $106(21)$ & $9(2)$ & $69(14)$ & $51(10)$ & $55(52 \%)$ & $48 \%$ \\
\hline
\end{tabular}

Words in bold print were counted as correct information units. 'PM' = per minute, 'WPM' = words per minute, ' $\mathrm{CIU}$ ' $=$ correct information units. 